\title{
RADIO EMISSION FROM STELLAR FLARES
}

\author{
JAN KUIJPERS
}

Sterrekundig Instituut, Utrecht University, P.O. Box 80000, 3508 TA Utrecht, The Netherlands

\begin{abstract}
An overview is given of the observations of stellar radio 'flares', defined as radio emission which is both variable in time and created by explosive releases of magnetic energy. The main sources of such flares are late-type Main-Sequence stars, classic close binaries, X-ray binaries, and pre-Main-Sequence stars.

We summarize the interpretations of these observations in terms of the various incoherent and coherent emission mechanisms. The possible importance of a coherent emission process in electrostatic double layers is pointed out.

We briefly indicate the diagnostic importance of radio emission for the flare process in classic and compact stars. In particular we discuss the possible production of radio flares from interactions between an accretion disk and the magnetic field of the central object.
\end{abstract}

\section{Introduction}

We define stellar radio flares as variable radio emission from stellar mass objects which is possibly produced by explosive releases of magnetic energy. Such flares have been found in (1) late-type Main-Sequence stars including the classic flare stars (dMe and dKe stars), (2) detached, semi-detached, and contact close binaries (RS CVn and, respectively, Algol-type and W UMa-type stars), (3) X-ray binaries, (4) pre-MainSequence stars (T Tauri and protostars), (5) magnetic Ap/Bp stars, and (6) late-type giants and supergiants (Hjellming and Gibson, 1980; Gibson, 1985). In this review we shall discuss the first four groups. For the recently discovered variability (time-scale of months) in Ap/Bp stars we refer to (Drake et al., 1987; André et al., 1988). A recent catalogue of the more extended group of (variable and nonvariable) continuum radio stars (but excluding pulsars, compact $\mathrm{H}$ II regions and supernovae) has been compiled by Wendker (Wendker, 1987). Recent reviews of radio stars in general including $\mathrm{O}, \mathrm{B}$ stars (bremsstrahlung, gyrosynchrotron, shocks), $\mathrm{M}$ giants, symbiotic stars are given in Dulk (1985, 1988), Kuijpers (1985b), Bookbinder (1988), and Hjellming (1988).

In general the observed radio emission from stellar flares (a few hundreds of $\mathrm{MHz}$ to a few tens of $\mathrm{GHz}$ ) is negligible in comparison to the emission at X-ray, UV, and optical frequencies. However, since part of the radio emission originates at the very site of the primary energy release, it serves as an important diagnostic tool for the study of the energy release and acceleration mechanisms in the flare. As in the case of solar flares this works best if the source is spatially resolved at the various frequencies and in both modes of circular polarization. The spatial extent, obtained from direct measurements or, in case of a fluctuating signal, indirectly from light-travel time estimates, and the flux determine the brightness temperature at that frequency and polarization, given by

$$
T_{b}(f) \simeq 4.8 \times 10^{10} \frac{S_{f}}{100 \mathrm{mJy}}\left(\frac{0.48 \mathrm{mas}}{\alpha} \frac{5 \mathrm{GHz}}{f}\right)^{2} \mathrm{~K},
$$


where the source has a circular projection of radius $\alpha$ in milliarcsec (mas) and a polarized (of the specific mode) flux density $S_{f}$ at frequency $f(1 \mathrm{mJy}=1$ millijansky $=$ $10^{-26} \mathrm{erg} \mathrm{cm}^{-2} \mathrm{~Hz}^{-1} \mathrm{~s}^{-1}$; the apparent radius of the Sun at a distance of $10 \mathrm{pc}$ is $\alpha=0.48$ mas). The brightness temperature together with polarization, temporal and spectral characteristics then forms an important constraint on the radiation mechanism and the distribution of the radiating particles in momentum space.

The only stellar flare sources other than the Sun in which spatial structure has been observed (with Very Long Baseline Interferometry), are the relatively strong ( $>100 \mathrm{mJy}$ ) and extended nearby (distance less than 100 parsec) classic close binaries (of the RS CVn and Algol type; angular size 1-3 milliarcsec) and jets of some X-ray binaries and pre-Main-Sequence objects (protostars and T Tauri stars). Recently detailed frequency information of radio flares from stars other than the Sun has become available in the form of multiple-frequency observations and so-called dynamic radio spectra (Bastian and Bookbinder, 1987).

The main observational problem of course is that the active flaring state in most known flaring objects is relatively short; for the classic flare stars the flare frequency is often much less than one per day; therefore, some courage is required to allocate time to observe stellar flares. However, such observations, and in particular when they are done simultaneously in the X-ray, ultraviolet, optical and infrared domain, can be of great value for the fascinating subject of explosive, magnetically controlled, energy release in astrophysics (Uchida, 1986).

\section{2. dMe Stars}

This is the group of the classic flare stars (dMe type) first studied by Lovell at radio frequencies (Lovell, 1971). The flaring activity of these stars is the most conspicuous of the entire group of active Main-Sequence stars with convective envelopes (spectral type later than $\mathrm{F} 2$; but most of which have not yet been detected in the radio (Bookbinder and Walter, 1988; Seaquist and Taylor, 1985; Willson, 1987; Bastian, Dulk, and Chanmugan, 1988), including our Sun with its comparatively weak flaring activity. The properties of the radio flares are summarized in Table $I$.

\subsection{CohERENT EMISSION PROCESSES}

Some of the burst emission is probably of coherent origin because of:

Narrow-band emission, in dynamic radio spectra with a relative frequency extent $<0.002$ (Bastian and Bookbinder, 1987) and <0.06 (Jackson, Kundu, and White, 1987); in multiple frequency observations with a relative bandwidth of a few percent or less (Lang and Willson, 1986a, 1988; White, Kundu, and Jackson, 1986; Willson, 1987).

High brightness temperature. Occasionally much larger radio luminosities than in Table I are observed for bursts which are often, but not always (Kundu and Shevgaonkar, 1988), highly circularly polarized (>75\% up to $100 \%$ ) (Gibson and Fisher, 1981; Gibson, 1984; Lang and Willson, 1988; Kundu et al., 1988; Jackson, Kundu, and White, 1987; Willson, 1988; White, Kundu, and Jackson, 1986; Kundu 


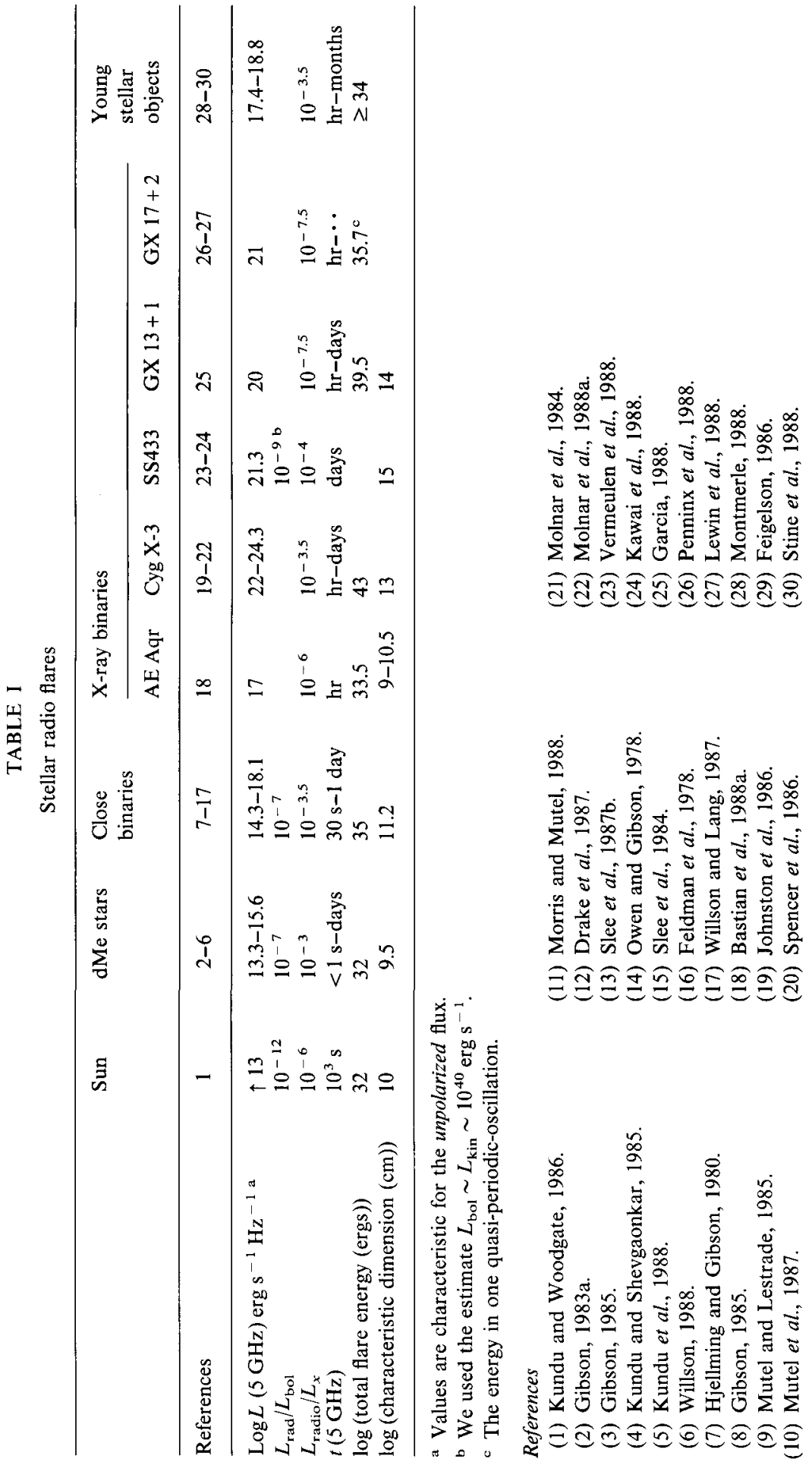


et al., 1987). Brightness temperatures are $7 \times 10^{12} / \beta^{2} \mathrm{~K}$ at $330 \mathrm{MHz}$ (Kundu and Shevgaonkar, 1988); $3 \times 10^{12} / \beta^{2} \mathrm{~K}$ at $408 \mathrm{MHz}$ (Davis et al., 1978); $1.8 \times 10^{11} / \beta^{2} \mathrm{~K}$ at $1.5 \mathrm{GHz}$ (Kundu et al., 1988); $8.5 \times 10^{9} / \beta^{2} \mathrm{~K}$ at $5 \mathrm{GHz}$ (Slee et al., 1981) ( $\beta$ is defined as the source linear dimension divided by twice the stellar radius). Sometimes strong temporal variability (down to $0.005 \mathrm{~s}$ (Lang and Willson, 1986b)) can be used to find an even more stringent limit to the brightness temperature: $10^{13} \mathrm{~K}$ at $1.4 \mathrm{GHz}$ taking the product of the observed $e$-folding time of $0.2 \mathrm{~s}$ and the speed of light as an upper limit to the source dimension (Lang et al., 1983) and $3.6 \times 10^{15} \mathrm{~K}$ (rise time $0.02 \mathrm{~s}$ ) at $430 \mathrm{MHz}$ (Bookbinder et al., 1989). Such brightness temperatures have also been observed in the case of the Sun at metric and decimetric wavelengths (Slottje, 1978; Benz, 1986; Dröge, 1967; Stähli and Magun, 1986).

Because of the strong polarization, the high brightness temperature and the narrow frequency extent, these bursts can only come from a coherent plasma process. (Note, however, that coherent radiation can be completely unpolarized and/or have a relatively large bandwidth.)

\subsubsection{Is the Electron Cyclotron Maser the Universal Explanation of Coherent Emission} from Flare Stars?

For a correct treatment of a linear plasma instability near a cyclotron resonance $\left(\omega-s \omega_{c e} / \gamma-k_{\|} v_{\|}=0\right)$ it is essential to take into account the relativistic dependence of the cyclotron frequency on the particle velocity (Blanken, Stix, and Kuckes, 1969; Zheleznyakov and Zlotnik, 1975). Following the early work on a direct instability of electromagnetic waves at (harmonics of) the electron-cyclotron frequency (Twiss, 1958; Ramaty, 1969; Melrose, 1973), an impressive amount of progress has been reached on the electron-cyclotron maser (Stepanov, 1978; Wu and Lee, 1979; Holman, Eichler, and Kundu, 1980; Melrose, Hewitt, and Dulk, 1984; White, Melrose, and Dulk, 1986; Aschwanden, Hewitt, and Dulk, 1989; McKean, Winglee, and Dulk, 1989), and its relativistic version (McCray, 1966; Zheleznyakov, 1967; Louarn, Le Quéau, and Roux, 1987), as an explanation for the above discussed strongly polarized bursts. This direct radiation instability occurs when energetic electrons are released in a magnetic flux tube (magnetic bottle) and develop a (one-sided) loss-cone distribution at the magnetic mirrors at the footpoints and if the ratio of electron-cyclotron frequency to plasmafrequency is sufficiently large (Winglee, Dulk, and Pritchett, 1988; larger than 3, or in the related synchrotron maser for MeV electrons larger than 0.3 (Louarn, Le Quéau, and Roux, 1989)). Since the surface coverage with kilogauss magnetic fields in flare stars is much larger than on the Sun (Saar and Linsky, 1985; Saar, 1988), the cyclotron maser probably occurs relatively often in flares on dMe stars. However, it is probably only one of several and not the universal coherent radiation process as is often suggested: In the weaker field regions (ratio of cyclotron to plasma frequency less than three) loss-cone distributions of trapped electrons do not directly produce radiation at one or twice the electron cyclotron frequency but cause the growth of upper hybrid waves which lead to radiation at once (Kuijpers, 1974, 1975; Zheleznyakov and Zlotnik, 1975; Kuijpers, van der Post, and Slottje, 1981) or twice (Stepanov, 1974; Winglee and Dulk, 1986) the 
upper hybrid frequency (Holman, 1983). Further, in the case of the Sun flares exhibit a rich variety of radio bursts (McLean and Labrum, 1985; Krüger, 1979) with different plasma instabilities. A large number of coherent radiation processes has been proposed for intense radiation observed from members of the solar system (see for a review, for example, Kuijpers, 1985a, b, 1980; Melrose, 1986; Papadopoulos and Freund, 1979; Tsytovich, 1973). Finally, from solar radio astronomy it is well-known that to determine the kind of burst reliably, in general dynamic spectra of high resolution both in frequency (down to $100 \mathrm{kHz}$ ) and time (down to millisec) are required. Therefore, although coherent emission processes certainly operate in flare stars, the existing identifications with specific processes, e.g., at the cyclotron frequency (White, Melrose, and Dulk, 1986), are at best tentative; often radiation at the upper hybrid frequency is equally well possible, as is observed in a class of solar bursts (the stationary type IVdm radiation from magnetic traps with continued injection (Kuijpers, 1980)).

Of course these high-frequency instabilities of loss-cone distributions for various ratios of cyclotron to plasma frequency form one physical family. Both the electroncyclotron maser and the upper hybrid instability are simply opposite extremes of the magnetically controlled phenomenon of trapped energetic particles. Observationally, the characteristic emitted frequency is expected to vary from once or twice the cyclotron frequency to the upper hybrid frequency as the ratio electron cyclotron to plasma frequency shifts from values larger than unity to values smaller than unity; therefore, for order of magnitude estimates the observed frequency can be put equal to the upper hybrid frequency.

\subsubsection{Coherent Emission from Electrostatic Double Layers}

We now briefly describe a possible coherent radiation mechanism from electrostatic double layers, which has not appeared in the literature before but which may be of potential importance for magnetic flares in various objects.

Electrostatic double layers were first proposed in an astrophysical context by Alfvén (Alfvén, 1958) and since then have been advocated in a variety of cosmic high-energy phenomena (Alfvén, 1981). The reason is that double layers have dynamic properties which are characteristic for explosive phenomena in the Universe: the acceleration of electrons, of ions and of mass motions. Electrostatic double layers are localized (dimension of 10-100 Debye lengths) electrostatic potential drops along the magnetic field. The structure is generated by charge separation, which is self-consistently maintained by the motion of particles accelerated in this potential drop (Raadu and Rasmussen, 1988). We restrict ourselves to strong double layers in which the potential drop corresponds to several times the thermal particle energy. They have been observed to occur in current carrying plasmas in the laboratory (Torvén, 1982; Hershkowitz, 1985; Torvén, Lindberg, and Carpenter, 1985). A substantial part of the global magnetic energy of the electric circuit is released locally in double layers and in a highly nonthermal manner. Therefore, they are of interest for astrophysical flares of magnetic origin: the potential drop accelerates particles of both charges and at the same time allows relaxation of the magnetic stresses by the local unwinding of the magnetic field (Jacobsen and Carlqvist, 
1964; Alfvén and Carlqvist, 1967; Raadu, 1984). Still many problems in our understanding of double layers remain to be solved, in particular on the question of their formation and on double layers of the explosive type. Under stellar, in contrast with laboratory, conditions it is not likely that the drift velocity of the current particles surpasses the thermal velocity. Rather an alternative may be local reconnection of magnetic fields which changes the path of an electric current system; because of the large dimension the current system has a huge inductance and a large inductive voltage is generated locally, perhaps leading to the (transient) formation of a double layer. In this case one should think of a spatially distributed large collection of many transient double layers (Hénoux, 1987).

Coherent radiation is expected to come from double layers at frequencies somewhat above the ambient plasma frequency (Kuijpers et al., 1989). The reason is that electrons are accelerated in the double layer and, therefore emit 'linear accelerator' radiation. The radiation is expected to be of the masering type somewhat similar to the free electron laser. Now, however, the beam is strongly inhomogeneous, not necessarily relativistic and instead of the periodic wiggle of the electron laser the spatial structure of the strong electrostatic potential of the double layer appears. The radiation should be strongest in directions perpendicular to the electric field: in a static structure the resonance condition $\left(\omega_{t}-\mathbf{k}_{t} \cdot \mathbf{v}=\omega-\mathbf{k} \cdot \mathbf{v}\right.$, where $\omega, \mathbf{k}$ are the frequency and wave vector of the electromagnetic (subscript $t$ ) and of the electrostatic (no subscript) wave) reduces to $\omega_{t}=-\mathrm{k} \cdot \mathrm{v}$ which for a double layer is of the order of the electron plasma frequency outside. Perhaps this mechanism can explain the short intense bursts of high-frequency radiation which is observed in disrupting double layers in the laboratory (Lindberg, 1987, 1988).

\subsection{SPECTRAL CORRELATION}

There is a lack of detailed correlation in time between emission in various frequency domains:

- Between X-ray (0.02-2 keV) and optical observations (Doyle et al., 1988). This could be caused by a flare occurring in a closed magnetic structure low in the chromosphere, or alternatively, by heating by a proton beam instead of an electron beam, inhibiting the rise of heated material ('evaporation') by the directed momentum of the proton beam and explaining the absence of microwave emission by the absence of energetic electrons (van den Oord, 1988).

- Between emission at different radio frequencies (Kundu and Shevgaonkar, 1988; Kundu and Hjellming, 1985). This may, however, be caused by a complex narrow-band structure as is observed for solar radio flares.

- Between soft X-rays (0.04-2 keV) and radio (6 and $20 \mathrm{~cm}$ ) observations (Kundu et al., 1988). This may be caused by a number of effects: (i) Observing at the wrong (too soft) X-ray frequency: on the Sun only the hard X-ray flare ( $>20 \mathrm{keV})$ and microwave $(1.8 \mathrm{~cm})$ time profiles are very similar (Dennis, 1988). (ii) Gyroresonance absorption, Razin suppression near the plasma frequency or free-free absorption; such effects have been demonstrated to be important for solar flares (Klein, 1987), where in one case the 
gyrosynchrotron radiation from energetic electrons responsible for a hard X-ray burst observed up to $200 \mathrm{keV}$ was negligible below $30 \mathrm{GHz}$ ! (iii) A column depth above the transition region larger than $1.7 \times 10^{20} \mathrm{~cm}^{-2}$ or a rapidly converging magnetic field (Lu and Petrosian, 1988). (iv) A variety of particle spectra: in some (impulsive) hard X-ray flares microwaves $(17 \mathrm{GHz})$ are emitted mainly by $<200 \mathrm{keV}$ electrons with a steeply decreasing energy spectrum and which precipitate in lower layer of high $(900 \mathrm{G})$ field strength; in other (extended) flares microwaves are emitted mainly by $\mathrm{MeV}$ electrons trapped in a coronal loop of relatively low $(<100 \mathrm{G})$ field strength, and the correlation is best for very hard ( $>360 \mathrm{keV}$ ) X-rays (Kosugi, Dennis, and Kai, 1988).

More case studies of flares from these stars with simultaneous coverage at many frequencies are required to show that the $\mathrm{X}$-ray and radio activity are not the combined result of the same flares (Holman, 1985) or that flares from dMe stars would be physically very different from solar flares.

\subsection{QUIESCENT EMISSION AND MICROFLARING}

Some flare stars show unpolarized or weakly polarized slowly varying (time-scale hour-days) emission or a background of such emission (Gary and Linsky, 1981; Linsky and Gary, 1983; Gary, 1985; Stewart et al., 1988). The radiation cannot be bremsstrahlung (because of the magnitude of the observed X-ray flux) and either gyroresonance emission of a thermal plasma or gyrosynchrotron emission is at play (Gary, 1985). The gyroresonance interpretation requires a large source size $\left(R=3 R_{*}\right.$, $\left.T=3 \times 10^{7} \mathrm{~K}, B=200 \mathrm{G}, n=2 \times 10^{8} \mathrm{~cm}^{-3}, s=10\right)$ and sometimes meets some difficulties: one-temperature model not adequate (Kundu and Shevgaonkar, 1985); precipitation losses are two orders of magnitude above the X-ray luminosity (Kundu et al., 1987). The gyrosynchrotron interpretation requires a less dense gas of higher particle energy over a smaller volume, either in the form of a tenuous high-temperature component or a non-thermal population $\left(R=R_{*}, T=2.7 \times 10^{8} \mathrm{~K}\right.$, $n=10^{5-6} \mathrm{~cm}^{-3}, B=300 \mathrm{G}$ ); it also requires less severe energy losses (precipitation losses $1.8 \times 10^{29} H \mathrm{erg} \mathrm{s}^{-1}$, where $H \ll 1$ is a filling factor for the regions of strong diffusion (Kundu et al., 1987)), and explains well the spectral shape of the quiescent emission from dMe stars (magnetic dispoles significantly smaller in size than a stellar radius; typical turnover frequency of the calculated spectra between 4-30 GHz (White, Kundu, and Jackson, 1989)).

To sustain this so-called quiescent emission the continued production of accelerated particles is required for several hours (Kundu et al., 1987; Willson, 1987) or once every hour (Kundu and Shevgaonkar, 1985). Then this leaves open the possibility that the radiation is plasma radiation (Holman, Bookbinder, and Golub, 1985) as in the case of a class of stationary solar metric continua (which can be highly polarized).

Further the distinction between this form of activity and flares becomes rather vague. It may well be that the quiescent emission is caused by a sequence of nanoflares (Schadee, de Jager, and Švestka, 1983; Schadee, 1986; Parker, 1988) or microflares (Lin et al., 1984; Athay, 1984; Canfield and Metcalf, 1987) as in the solar case. Evidence for stellar microflares exist (Butler et al., 1986; Whitehouse, 1985), occurring over tens 
of seconds to ten minutes with individual energies of $2 \times 10^{30} \mathrm{erg}$ (van den Oord and Barstow, 1988) up to $5 \times 10^{30}-10^{31} \mathrm{erg}$ (Kundu et al., 1988).

\subsection{Stable Magnetic structures?}

For $\mathrm{YZ} \mathrm{CMi} \mathrm{all} \mathrm{observations} \mathrm{at} \mathrm{various} \mathrm{frequencies} \mathrm{show} \mathrm{the} \mathrm{same} \mathrm{sense} \mathrm{of} \mathrm{polarization}$ (Kundu and Shevgaonkar, 1988). Possibly this implies the existence of asymmetric leading-trailing stellar spots and a stable orientation (and a viewing angle well out of the magnetic equator) over the observed number of years (Gibson, 1983b).

\subsection{THE SOLAR CONNECTION}

The main observational difference then between solar and dMe flares is at present only the much larger flare luminosities and total energies for these stellar flares. Theoretically this is also expected if the flares are of magnetic origin and if the magnetic energy content of the stellar coronae are much larger than for the Sun (Kuijpers, 1985b). For the latter substantial evidence exists in the form of observed strong surface fields $(\mathrm{kG})$ with large filling factors (Saar, 1988).

\section{Close Binaries}

About 100 close binaries (1-30 days period) of the RS CVn (detached) and Algol (semi-detached) type have been detected at radio frequencies (see Table I). It now appears that the radio luminosity $(8 \mathrm{GHz})$ is clearly correlated with the rotation rate (Stewart et al., 1988). The spectrum sometimes peaks between 1-15 GHz (Mutel et al., 1987). For individual sources the spectral index between $1.5-5 \mathrm{GHz}\left(S_{f} \sim f^{\alpha}\right)$ increases with radio luminosity from -1 to +1 (Mutel et al., 1987). Usually the degree of circular polarization is between 0 and $30 \%$, but sometimes higher. For some, non-eclipsing, systems the sense of circular polarization ('helicity') reverses between 1.4 and $4.9 \mathrm{GHz}$ (Mutel et al., 1987). For individual systems the fractional circular polarization decreases with increasing luminosity at $5 \mathrm{GHz}$ (Mutel et al., 1987).

A few contact binaries (W UMa systems) have been detected in the radio: a flare in VW Ceph showed radio emission arriving a few minutes before the soft X-rays, very similar to the solar flare case (Vilhu et al., 1988); FK Comae Berenices has been detected at 2,6, and $20 \mathrm{~cm}$ and the emission requires accelerated electrons (Hughes and McLean, 1987).

\subsection{Core-halo gyrosynchrotron SOURCE}

Dual-frequency VLBI of several of these sources (Lestrade, 1988; Mutel et al., 1985; Lestrade et al., 1988) shows the presence of a variable core-halo structure. For the best measurements the core is smaller than the $\mathrm{K}$-subgiant (assumed to be the active star, see figure in Mutel et al., 1985) and the corresponding brightness temperature at $5 \mathrm{GHz}$ is at least $1-3 \times 10^{10} \mathrm{~K}$, requiring electron kinetic energies of $1.3-3.9 \mathrm{MeV}$ in the case of incoherent emission; the size of the halo is comparable to the dimension of the binary $\left(10^{12} \mathrm{~cm}\right)$ and the corresponding brightness temperature of the halo at $5 \mathrm{GHz}$ is typically $(5-10) \times 10^{8} \mathrm{~K}$. The observed brightness temperatures, dimensions, spectral index, and frequency turnover, circular polarization and helicity reversal are best explained as 
gyrosynchrotron emission of electrons with Lorentz factors 2-10 in magnetic fields of strengths 30-200 G (Owen, Jones, and Gibson, 1976; Spangler, Owen, and Hulse, 1977; Spangler, 1977; Feldman, 1983; Borghi and Chiuderi-Drago, 1985; Kuijpers and van der Hulst, 1985; Lestrade et al., 1988). The characteristic frequency dependence of the source size agrees well with models of gyrosynchrotron emission from inhomogeneous distributions in magnetic loops in close binaries (Borghi and Chiuderi-Drago, 1985; Klein and Chiuderi-Drago, 1987; Lestrade et al., 1988). The radiation from the core is self-absorbed and unpolarized gyrosynchrotron emission in contrast with the optically thin radiation from the halo. As the luminosity increases the degree of circular polarization decreases, essentially since the luminosity increase is coming from the self-absorbed core (Mutel et al., 1987).

\subsection{Continuous acCEleration}

When VLBI observations are absent a flare is traditionally modelled by an expanding, initially optically thick, gyro-synchrotron source (Mutel et al., 1985). In this model the emission becomes optically thin, and the maximum flux density decreases, at progressively lower frequencies as the blobs expand. In the case of a radio flare from Algol, however, dual-frequency VLBI observation (Lestrade et al., 1988; Lestrade, 1988) showed the flux increase not to be caused by expansion of an energetic cloud or loop, but by an increase in brightness temperature of a source with dimension three times the sub-giant radius (which went up to $3 \times 10^{10} \mathrm{~K}$ at $8.4 \mathrm{GHz}$ ) and the continued production of high-energy electrons. Such an acceleration of electrons occurring over prolonged periods of time of order hours or more was already indicated by the general radio flux behaviour in time of these sources, e.g., Kuijpers and van der Hulst (1985).

\subsection{Coherent EMISSION}

(Gyro)synchrotron emission, however, cannot always be the cause of the observed emission. Occasionally a highly polarized (Mutel and Weisberg, 1978) intense outburst or variations on the time-scales of minutes (Brown and Crane, 1979; Newell et al., 1980; Mutel et al., 1987; Lestrade et al., 1988; Willson and Lang, 1987) have been observed. The only explanation for these cases of highly circularly polarized and intense bursts is some form of coherent or 'plasma' radiation as in (Melrose and Dulk, 1982); see Section 2.1 .

\subsection{SPECTRAL CORRELATION}

As is the case for $\mathrm{dMe}$ stars there is an absence of detailed correlation between activity at different frequencies: an ultraviolet flare with no radio $(1.5$ and $4.9 \mathrm{GHz})$ emission (Lang and Willson, 1988); activity in X-rays and in the radio with non-correlated variations (White, Kundu, and Jackson, 1986; van den Oord et al., 1989). Because this lack of detailed correlation can easily be explained by a variety of effects (see Section 2.2), we conclude that energetically it seems likely that the activity is causally related. 


\subsection{Global magnetic Structures}

That large magnetic structures on these stars can exist over a long duration follows from the sense of circular polarization and the helicity reversal between $1.4-5 \mathrm{GHz}$, which, for two RS CVn systems, stays the same over ten years (Mutel et al., 1987). Because of their small separation (5-10 stellar radii) the binary components are tidally locked and rotate nearly synchronously with the orbital motion, they have high rotational velocities (periods of the order of a few days) and, if they also have a convective envelope, both stars probably have a dynamo magnetic field. Thus it is not surprising if magnetic structures would exist which connect both stars ('magnetospheres'). Such is also indicated by the observed core-halo structure. Further, the observed radio emission shows no clear dependence on phase, indicating that the halo extends over much more than a stellar radius out of the orbital plane. Finally the mean circular polarization in a few binaries increases from 0 to $30 \%$ as the angle of the orbital plane with the line of sight varies from $0^{\circ}$ to $90^{\circ}$ (Mutel et al., 1987); again this points to a large-scale magnetic structure which has some symmetry about the orbital plane.

\subsection{DUPLICITY-ENHANCED ACTIVITY}

Even if the stellar rotation is synchronous with the orbit, one still expects violent relaxations between the individual evolving stellar magnetic fields (Bahcall, Rosenbluth, and Kulsrud, 1973; DeCampli and Baliunas, 1979; Simon, Linsky, and Schiffer, 1980; Uchida and Sakurai, 1983; Lamb et al., 1983; van den Oord, 1988). The extended halo sources with dimensions comparable to the separation of the binary constitute the observational evidence that such interactions are important. Further since in Algol, which is a semi-detached system with transient accretion gas rings (Kaitchuk and Park, 1988), an X-ray flare and radio outbursts have been observed very similar to those in RS CVn (detached) systems, this perhaps means that accretion also leads to driven magnetic reconnection.

\subsection{ENERGY BUDGET}

The required rate of energy injection in relativistic particles is of course dependent on the assumed radiation process and on source conditions such as magnetic field strength and background density. For $\sigma^{2} \mathrm{CrB}$ the energy in high-energy electrons in the synchrotron interpretation (Lorentz factor 3, magnetic field strength $35 \mathrm{G}$ ) is $10^{30} \mathrm{erg}$, which is a fraction $10^{-6}$ of the energy in the X-ray gas (Kuijpers and van der Hulst, 1985). Both in the case of $\sigma^{2} \mathrm{CrB}$ (Kuijpers and van der Hulst, 1985) and of Algol (van den Oord et al., 1989) the mildly relativistic electrons responsible for the gyrosynchrotron emission could be considered as the high-energy end of an initially cospatial power-law distribution emitting the X-rays; the fraction of the energy put into the particles with energies above $10 \mathrm{keV}$ would be only $5 \times 10^{-2}$ for Algol.

On the basis of the observed core-halo structures, their temporal variability and the energetics of the radio and X-ray emission we, therefore, adopt the working hypothesis that the radio emitting electrons are accelerated at the same time as the $X$-ray gas is energized. This can occur in two ways: 
(1) In the form of strong and impulsive flares as seen in X-rays with dimensions smaller than a stellar radius: total energy in X-rays of $2.4 \times 10^{34}-1.0 \times 10^{35} \mathrm{erg}$, from loops of length $4.5 \times 10^{10}-1.6 \times 10^{11} \mathrm{~cm} \quad(=0.3-0.6$ stellar radius $)$ and total volume $6.9 \times 10^{29}-1.4 \times 10^{31} \mathrm{~cm}^{3}$ and density $6 \times 10^{11}-3.1 \times 10^{11} \mathrm{~cm}^{-3}$ (van den Oord, Mewe, and Brinkman, 1989; van den Oord and Mewe, 1988). In this case the highenergy particles must be able to escape to an extended region, comparable to the binary separation (Massi et al., 1989), and of low density in view of variations in the radio flux at $1.4 \mathrm{GHz}$ of the order of one day (background density $<10^{7} \mathrm{~cm}^{-3}$ for $\sigma^{2} \mathrm{CrB}$ (Kuijpers and van der Hulst, 1985)).

(2) On a more gradual scale in the form of many local reconnections of magnetic field in between the binary components.

The large flare energy in these classic close binaries as compared to dMe stellar flares, which again are much larger than solar flares, can be understood if they are all of magnetic origin: in the case of $\mathrm{dMe}$ stars and of classic close binaries the filling factor of photospheric magnetic fields is large compared to the solar case; further for similar photospheric field strengths the coronal volume, and, therefore, also the magnetic energy, is much larger for classic close binaries than for dMe stars.

\section{X-Ray Binaries}

A small number of X-ray binaries with compact stellar objects show strong and variable radio emission: 13 sources at $5 \mathrm{GHz}$ out of $117 \mathrm{X}$-ray sources (Nelson and Spencer, 1988); see the tables in Hjellming and Johnston (1985) and Chanmugan (1987). Since the spectral index of the observed flux varies between +1 and -1 and the brightness temperatures estimated from the observed time variability are moderate (e.g., $10^{6.7} \mathrm{~K}$ at $5 \mathrm{GHz}$ and $10^{9.1} \mathrm{~K}$ at $1.5 \mathrm{GHz}$ for $\mathrm{GX} 13+1$ (Garcia et al., 1988); $10^{9}-10^{12} \mathrm{~K}$ at $5 \mathrm{GHz}$ for the cataclysmic variable AE Aqr (Bastian, Dulk, and Chanmugan, 1988a)) the radio emission is usually ascribed to the (gyro) synchrotron process (Dulk, 1986).

The white dwarf binaries (cataclysmic variables) detected in the radio are magnetic cataclysmic variables (coherent pulsations in the optical light curve; strong magnetic field guiding the accreting mass near magnetic poles on the white dwarf), dwarf novae (optical outbursts in a disk around the white dwarf), novae, and recurrent novae. We shall not discuss (recurrent) novae since their radio emission is probably not caused by the release of magnetic energy (Chanmugan, 1987; Bookbinder and Lamb, 1987) (bremsstrahlung or (gyro/synchrotron radiation from shock accelerated electrons due to the ejected gas).

\subsection{LOCATION OF CENTRAL RADIO EMISSION}

A small source can be detected only if its brightness temperature is large enough. Further, radio signals of too low frequencies cannot propagate in dense magnetoplasma near the compact object or are substantially absorbed in intervening gas. As a result radio observations are a diagnostic probe of the outer regions only. Below we shall use field strengths estimated from assumed magnetic dipoles at the location of the compact objects; estimates for gas densities are based on hypothetical isothermal and isotropic 
mass loss rates, although of course in reality the distribution of the gas around X-ray binaries is very inhomogeneous, with accretion disks, jets and streams. The following physical effects are important:

(1) Brightness temperature: an unpolarized source at a distance $d$ and brightness temperature $T_{b}$ at frequency $f$ produces a flux $S$ at frequency $f$ it its radius exceeds

$$
R=3.1 \times 10^{10}\left(\frac{S}{1 \mathrm{mJy}}\right)^{1 / 2}\left(\frac{f}{10^{9} \mathrm{~Hz}}\right)^{-1}\left(\frac{T_{b}}{10^{12} \mathrm{~K}}\right)^{-1 / 2} \frac{d}{100 \mathrm{pc}} \mathrm{cm} .
$$

Note that the brightness temperature is not limited by the conventional inverse Compton limit of $10^{12} \mathrm{~K}$ if the radiation is not synchrotron radiation.

(2) Electron plasma frequency: radiation can only escape above the electron plasma frequency. A location at a distance $r$ from the central object with a wind (or jet) $\dot{M}$ with velocity $v$ is visible only above a frequency

$$
f>4.5\left(\frac{\dot{M}_{-7}}{v_{8}}\right)^{1 / 2} r_{11}^{-1} \mathrm{GHz} \text {. }
$$

Here $\dot{M}_{-7}$ is the mass loss rate in units of $10^{-7} M_{0} \mathrm{yr}^{-1}, v_{8}$ the velocity in units of $10^{8} \mathrm{~cm} \mathrm{~s}^{-1}$ and $r_{11}$ the radius in units of $10^{11} \mathrm{~cm}$. The observed expansion speed is of order of the escape velocity and varies from a few thousand $\mathrm{km} \mathrm{s}^{-1}$ for a white dwarf to a hundred thousand $\mathrm{km} \mathrm{s}^{-1}$ for a neutron star. The mass loss and mass accretion rates vary considerably even within the same group of compact objects. From Equations (2) and (3) it is clear that in general only the outer regions of X-ray binaries $\left(>10^{10} \mathrm{~cm}\right)$ can be probed at radio frequencies, and at best in the far infrared; the inner regions can be detected in the radio only if both a (coherent) mechanism is at play with brightness temperatures substantially above $10^{12} \mathrm{~K}$ and if the central region is not obscured by disk or jet.

(3) Cyclotron frequency: the presence of a magnetic field prevents the escape of radiation below the electron-cyclotron frequency, and causes severe absorption of the escaping radiation in the overlying layers due to cyclotron resonances at the lower harmonics of the cyclotron frequency, $s f_{c e}$. This absorption increases with the electron density and the temperature of the overlying gas and the magnetic scale height (van den Oord et al., 1989). Estimating the magnetic field for an assumed dipole at the center of the compact object with polar field strength $B_{0}$ at radius $R_{0}$ (magnetic moment $0.5 B_{0} R_{0}^{3}$ ), a magnetic neutron star with $B_{0}=10^{12} \mathrm{G}$ and $R_{0}=10^{6} \mathrm{~cm}$ can only be observed at frequencies above

$$
f>2.8 r_{9}^{-3} s \mathrm{GHz},
$$

whereas a magnetic white dwarf with $B_{0}=10^{6} \mathrm{G}$ and $R_{0}=10^{9} \mathrm{~cm}$, can only be observed above

$$
f>2.8 r_{10}^{-3} s \mathrm{GHz} \text {. }
$$

(4) Free-free absorption: an estimate of the optical thickness from radius $r$ to infinity is obtained from $\tau=n_{e}^{2} k L$, where the electron density is, as before, derived from a wind 
with constant speed, the length is approximated by the density scale height, $L=r / 2$, and the expression for the absorption coefficient is taken from Mezger and Henderson (1967) with $a(f, T)=1$,

$$
\tau_{f f}=0.35\left(\frac{\dot{M}_{-7}}{v_{8}}\right)^{2} f_{9}^{-2.1} T_{4}^{-1.35} r_{14}^{-3}
$$

Free-free absorption prohibits the mapping of the inner regions of an X-ray binary in the radio and infrared for values $\dot{M}_{-7} / v_{8} \geq 10^{-3}$ unless the circumstellar mass distribution is extremely dependent on angle.

(5) Scattering: there are two effects: scattering by single electrons and by electron inhomogeneities.

Structural details are smeared out by free electron scattering. The optical thickness is

$$
\tau_{e s}=0.85 \frac{\dot{M}_{-7}}{v_{8}} r_{9}^{-1}
$$

Scattering by inhomogeneities along the line of sight are particularly important for sources in the galactic plane (Rickett, 1977). The change of the refractive index increases with the square of the wavelength. For Cyg X-3 at $10 \mathrm{kpc}$ the scattering size at $23 \mathrm{GHz}$ is estimated to be as large as 0.7 mas (Molnar, Reid, and Grindlay, 1988a), corresponding to a linear scale of $10^{14} \mathrm{~cm}$ at that distance.

From these effects we conclude that free-free absorption of the overlying gas or scattering by electron density inhomogeneities along the line of sight probably puts the most stringent lower limit on the structural details which can be observed at radio frequencies near the compact companion in a binary. Coherent radiation at far infrared wavelengths from the inner regions may be observable if not obscured by disk or jet.

\subsection{FLARE ORIGIN IN CATACLYSMIC VARIABLES}

Radio flares have been observed in three cataclysmic variables: AM Her (Bastian, Dulk, and Chanmugan, 1985), AE Aqr (Bookbinder and Lamb, 1987; Bastian, Dulk, and Chanmugan, 1988), and AO Psc (Wendker, 1987). Probably the radio flares are caused by accretion since their intensity is large compared to the radio flares of single late-type Main-Sequence dwarfs or compared to the weak radio emission from the cataclysmic variable progenitor V471 Tau (Bastian, Dulk, and Chanmugan, 1988). Further, only gravitational and not nuclear energy liberation plays a role, as any nuclear burning on the surface of the white dwarf in AM Her proceeds in a stationary non-impulsive manner. As the observed radio active cataclysmic variables are magnetic, probably also magnetic fields are important. AE Aqr has a relatively small field $\left(10^{5}-10^{6} \mathrm{G}\right)$ allowing the formation of an accretion disk and the flares possibly result from interactions between the white dwarf magnetic field and the disk (see Section 4.8 and Bookbinder and Lamb (1987)). AM Her, on the other hand, also has a magnetic field but of sufficient strength ( $\mathrm{few} \times 10^{7} \mathrm{G}$ at the white dwarf surface) that no accretion disk forms (Lamb 
and Melia, 1986). Its radio emission sometimes shows appreciable circular polarization $(25 \%)$ and is explained as gyrosynchrotron emission with a source size of radius $10^{11} \mathrm{~cm}$ (electrons with average Lorentz factor 2 in a magnetic field of $40 \mathrm{G}$; brightness temperature at $4.9 \mathrm{GHz}$ of $3 \times 10^{9} \mathrm{~K}$; harmonic number 40) (Chanmugan and Dulk, 1982; Bastian, Dulk and Chanmugan, 1985). The presence of polarization implies the existence of a magnetic field on a scale comparable to the size of the orbit and the flaring is perhaps caused by distortion of the white dwarf magnetic field in the outer region by the accreting material and subsequent magnetic reconnection with the field of the Main-Sequence companion which initially permeates the accreting material. Why no radio emission has been detected from all other magnetic cataclysmic variables is a problem, but it may be related to obscuration (Fürst et al., 1986; see Section 4.1).

\subsection{Coherent EMission}

In the cataclysmic variable AM Her a strong $\left(T_{b}>10^{10} \mathrm{~K}\right.$ at $\left.4.9 \mathrm{GHz}\right)$ and probably completely circularly polarized radio flare must have been produced by a coherent process (Dulk, Bastian, and Chanmugan, 1983).

In some dwarf novae radio emission has been discovered, with one to two days delay after the optical maximum (Benz and Güdel, 1989). The authors propose that the radiation is coherent cyclotron emission from electrons accelerated near the disk and radiating above the poles of the white dwarf (1000-2000 G).

Infrared (2.0-2.4 $\mu \mathrm{m})$ flare events of few minutes duration in Cyg X-3 without clear counterparts in X-rays (1.5-10.0 keV) (Mason, Cordova, and White, 1986), are interpreted by these authors as free-free emission from hot clumps ejected from the system in jets and forming the precursors of radio flares (typical parameters of a blob: $10^{11} \mathrm{~cm}$, $n=10^{14} \mathrm{~cm}^{-3}$ and $T=10^{6} \mathrm{~K}$, as compared to small radio flares with a dimension of $6 \times 10^{12} \mathrm{~cm}$ (Molnar, Reid, and Grindlay, 1984))

We would like to suggest an alternative of coherent emission in the infrared produced by energetic particles at early stages of the flares.

\subsection{EXPANDING SYNCHROTRON BLOBS OR SUSTAINED PARTICLE ACCELERATION}

In some of the X-ray binaries the observed temporal and spectral behaviour can be well described as gyrosynchrotron emission from relativistic electrons in expanding plasmoids mixed with nonrelativistic gas (Shklovski, 1965; van der Laan, 1966; Kellermann and Pauliny-Toth, 1968) (see also Section 3.2). In particular the model works well for the giant flares (reaching $20 \mathrm{Jy}$ ) (Gregory et al., 1972; Seaquist and Gregory, 1977) and the low-state radio flares (Molnar, Reid, and Grindlay, 1984) in Cyg X-3. However, this model is not valid for all sources. Even in the case of the giant outbursts from Cyg X-3 the flux density can vary by factors of three over a time-scale of hours to days (Johnston et al., 1986) indicating that several repeated injections of energetic particles contribute to the large expanding plasmoid. In the case of GX 13+1 (Garcia et al., 1988) and of Sco-X1 the radio outbursts show no systematic time delay at 6 and $20 \mathrm{~cm}$ wavelengths nor does the flux density reach higher values at higher frequencies, as expected in the expanding plasmoid model. In these sources continued production of relativistic particles 
is required. For a particular striking flare radio event in AE Aqr the initial size of the cloud in this model is at least $5 \times 10^{10} \mathrm{~cm}$ or, alternatively, the fast particles are injected over a longer period of time (Bastian, Dulk, and Chanmugan, 1988).

\subsection{JETS}

Some of the supposed neutron star binaries show radio jets (SS433, Cyg X-3, Sco X-1). In the case of SS433 the jets consist of individual knots travelling at constant speed of $0.26 c$ along a precessing axis (twin corkscrews) (Hjellming and Johnston, 1985; Romney et al., 1987; Vermeulen et al., 1988). The central source is unresolved $\left(<10^{15} \mathrm{~cm}\right)$ with brightenings which are not always associated with outgoing blobs. These individual blobs attain their maximum radio brightness at a distance of a few $10^{15} \mathrm{~cm}$ from the nucleus, and subsequently fade away on a time-scale of days as the knots travel outwards; the radio spectral index is always between -0.6 and -0.8 and indicates optically thin synchrotron emission. The observed brightenings in time and the discrete nature of the radio structures require repetitive flare-like production of relativistic particles; these properties are not explained by the 'cylindrical supernova' model of Hjellming and Johnston (1988). Cyg X-3 shows some similarities to SS 433: LBI observations of giant ( $10 \mathrm{Jy}$ at $5 \mathrm{GHz}$; decay time of days) radio outbursts can be interpreted as expanding double sources (projected bulk velocities of $0.35 c$ at a distance of $10 \mathrm{kpc}$ ) (Spencer et al., 1986); VLBI observations of two moderate (1 Jy at $23 \mathrm{GHz}$; decay time of hours) flares can be interpreted as an expanding and decaying double source (projected bulk velocities of $0.16 c-0.31 c$ at a distance of $10 \mathrm{kpc}$ ) (Molnar, Reid, and Grindlay, 1988a). Again in this source repeated injection of accelerated particles (see Section 4.4) is required.

\subsection{Correlated X-RAY and Radio FluX}

In some of the radio emitting X-ray binaries, Cir X-1 (Haynes, Lerche, and Murdin, 1980), LSI $+61^{\circ} 303$ (Taylor and Gregory, 1984), Cyg X-3 (Molnar, Reid, and Grindlay, 1984,1985 ) the radio flares are correlated with the X-ray emission, which is modulated on an orbital time-scale either because of variable mass transfer (Band and Grindlay, 1984 ) or a periodically varying viewing geometry.

In other sources, GX $13+1$ (Garcia et al., 1988) and Sco X-1, no evidence for a correlated X-ray and radio flux is found. It is noteworthy, however, that both objects show a bimodal hardness ratio diagram in X-rays, and that, at least for Sco X-1, radio flares occur only when the source is on the less luminous branch (Priedhorsky et al., 1986). Recently also GX $17+2$ has been found to emit strong radio emission when its $\mathrm{X}$-ray state is on the hard non-X-ray-flaring branch (Penninx et al., 1988). These systems all have quasi-periodic oscillations in X-rays and are believed to be low mass $\mathrm{X}$-ray binaries radiating at the Eddington limit. The observed absence of correlation can be understood in principle if the central object is a fast spinning weakly magnetized neutron star which acts as a flywheel, temporally storing accretion energy (Priedhorsky, 1986). At high mass accretion rates the luminosity is dominated by X-rays from accretion; at low-mass accretion rates the luminosity is supplied electrodynamically 
from interaction between the central object and the disk. Only in the latter case are important radio flares to be expected (Aly and Kuijpers, 1989).

\subsection{ENERGY BUDGET}

To explain the radio emission of GX $13+1$ in the synchrotron interpretation the required energy injection rate in relativistic electrons is of order $10^{36} \mathrm{erg} \mathrm{s}^{-1}$ (or $10^{-2}$ times the X-ray luminosity) for the optically thin, minimum energy case (Garcia et al., 1988); the equipartition field strength is $1 \mathrm{G}$.

For the cataclysmic variable AE Aqr the averaged required energy injection into relativistic electrons is of order one to a few times $10^{30} \mathrm{erg} \mathrm{s}^{-1}$ (or $10^{-3}$ of the bolometric luminosity of the system) at a field strength of $25 \mathrm{G}$ (Bastian, Dulk, and Chanmugan, 1988).

Characteristically less than one percent of the accretion energy is required for particle acceleration.

\subsection{A PERIODIC FLARE MODEL}

A powerful flare mechanism for disk accretion is the formation of magnetic links between the compact object (or in case of a central black hole: between a region near the horizon) and the disk (Aly and Kuijpers, 1989). If the magnetic field in the disk is highly non-uniform as is observed for the solar photosphere and for the galactic disk, the interaction between central field and disk field is expected to take place in the disk corona by stochastic reconnections leading to the formation of magnetic links. Since, in general, the footpoints of such a link rotate with different angular speeds kinetic energy is transformed electrodynamically into the field structure of the link. If the Alfven travel time along the coronal link is short in comparison with the shearing time the link evolves through a series of force-free equilibria, expands away from the disk and is expected to cause a flare, similar to the solar case (Aly, 1985; Low, 1986). When the outer footpoint is outside the corotation radius the energy is extracted from the rotation of the central object; if on the other hand the link is within the corotation radius (which occurs for small accretion rates) the energy is delivered by the accreting material. In the former case accretion is hindered, so that the magnetic flare energy is important, while in the latter case the link promotes accretion, so that the luminosity from the flare is relatively less important. The total luminosity from these magnetic flares in radiation, particle acceleration and explosive motion is on average (Aly and Kuijpers, 1989)

$$
L_{m}=1.3 \times 10^{35} B_{10}^{2} R_{* 6}^{6} R_{7}^{-4} l_{6} \frac{\Omega_{B}}{200 \mathrm{~Hz}} \mathrm{erg} \mathrm{s}^{-1},
$$

where $B_{10}$ is the polar field strength at the star in units of $10^{10} \mathrm{G}, R_{* 6}$ is the stellar radius in units of $10^{6} \mathrm{~cm}, R_{7}$ the distance of the outer footpoint in $10^{7} \mathrm{~cm}, l_{6}$ the thickness of the link in $10^{6} \mathrm{~cm}$ and $\Omega_{B}$ is the angular beat frequency between both footpoints. Equation (8) shows that in principle this process can release the required acceleration energies for a magnetized neutron star and, adapting Equation (8) for a white dwarf, also for a magnetic cataclysmic variable. 
Finally we like to point out that this linking process leads to a quasi-periodic production of flares on a period determined by the beat period.

\section{Young Stellar Objects}

Young stellar (or pre-Main Sequence) objects of low-mass show strong flaring activity. Depending on their infrared emission in the band $1-100 \mu \mathrm{m}$ they form a sequence characterized by the progressive disappearance of circumstellar dust and the optical appearance of the central object (T Tauri, FU Ori, and post-T Tauri stars) (Lada, 1988; Adams, Lada, and Shu, 1987); this sequence probably corresponds to an evolutionary succession, whereby the gas and dust envelope of the protostar, and ultimately also the gaseous disk, disappears. T Tauri and FU Ori stars have flares and strong variability ( $10^{34}$ ergs in 10 minutes) in the optical (Gahm, 1986, 1988). The variability of T Tauri stars is caused by a combination of variable obscuration by circumstellar dust, gas infall and the presence of $a$ disk and boundary layer (Lynden-Bell and Pringle, 1974; Brown, 1988; Bertout, Basri, and Bouvier, 1988). The strong flares in FU Ori objects are probably the result of enhanced mass accretion in a disk (Kenyon, Hartmann, and Hewett, 1988; Hartmann and Kenyon, 1988). The cause of the enhanced mass accretion is, however, not clear and may well be related to magnetic interactions between disk and star as in X-ray binaries (see Section 4.8).

\subsection{RADIO EMISSION}

Dramatic eruptions in X-rays $\left(>10^{34} \mathrm{erg}, 4 \times 10^{31} \mathrm{erg} \mathrm{s}^{-1}\right)$ in the Rho Oph dark cloud focused attention on young stellar objects as flaring stars (Montmerle, 1988; Feigelson, 1986). Subsequent radio observations of this and other dark clouds revealed the existence of stellar flaring objects in the radio (André, Montmerle, and Feigelson, 1987; André, 1986; Brown, 1987; Montmerle and André, 1988; Stine et al., 1988). The time-scales of the variable strong radio emission are so short (hours) that the radiation cannot be explained as free-free radiation from circumstellar gas (with corresponding time-scales of months to years). The radio flare fluxes (up to $1.5 \times 10^{18} \mathrm{erg} \mathrm{s}^{-1} \mathrm{~Hz}^{-1}$ ) are comparable to those of the RS CVn systems; it is possible that their quiescent emission is usually not observed as they are at a larger distance $(>130 \mathrm{pc})$ than the observed RS CVn stars (30-50 pc). Most ( $>60 \%$ ) have substantial X-ray emission but the reverse is not true (André, Montmerle, and Feigelson, 1987). In a radio survey of $\mathrm{T}$ Tauri stars a low detection rate was found and a strong correlation with sources with outflow (Cohen, 1987; Bieging, Cohen, and Schwartz, 1984; Cohen and Bieging, 1986). This suggests that the strong radio emitters are older than deeply embedded infrared sources, but younger than classical $\mathrm{T}$ Tauri stars.

A particularly interesting example of such a radio flaring object is the K0 pre-MainSequence X-ray star DoAr 21; its variable radio emission can be modelled with gyrosynchrotron radiation from a magnetic loop extending from one to ten stellar radii, a field strength of $1.5 \times 10^{2} \mathrm{G}$ at the base and $8 \times 10^{-2} \mathrm{G}$ at the top, a power-law electron energy spectrum with index 2 , a high-energy cut-off at $4.5 \mathrm{MeV}$ and a column density 
of energetic particles of $3.4 \times 10^{17} \mathrm{~cm}^{-2}$ (Feigelson and Montmerle, 1985; André, 1986).

\subsection{JETS}

Young stellar objects are often associated with jets: Many infrared sources have bipolar outflows (Lada, 1988); Some T Tauri stars have highly collimated visual jets (Bührke, Brugel, and Mundt, 1986; Mundt, 1988). The few T Tauri stars that are radio emitters are relatively strong and often associated with jet-like structures: These $\mathrm{T}$ Tauri stars, which are the least luminous young stellar objects, produce the largest fraction of radio to total luminosity (Hamann, Simon, and Ridgway, 1988). One object, DG Tau, shows a time-variable $5-15 \mathrm{GHz}$ structure with a bipolar morphology pointing towards a nearby Herbig-Haro knot $\left(200 \mathrm{~km} \mathrm{~s}^{-1}, 10^{2}-10^{4} \mathrm{~cm}^{-3}\right)$ (Cohen, 1987) in the optical jet (Cohen and Bieging, 1986). Another object, L1551, shows two unresolved radio components perpendicular to an extended elongated radio source (Bieging and Cohen, 1985 ) and possibly corresponding to an ionized inner part of a confining torus with projected radius of $25 \mathrm{AU}$ (Rodríguez et al., 1986). A similar radio structure of two point-like sources and two extended radio lobes is centered on the deeply embedded infrared source IRS 7 (Brown, 1988).

Since T Tauri stars sometimes have magnetic fields with a large surface filling factor, it is quite well possible that the flaring behaviour of these and younger stars is caused by magnetic interactions between star and disk.

\section{Conclusion}

Increasing evidence exists that at least some of the violent radio outbursts in dMe stars, classic close binaries, $\mathrm{T}$ Tauri stars and their predecessors, magnetic cataclysmic variables and luminous X-ray binaries are the result of magnetic flaring activity. The connection with solar flares is that the outbursts probably originate in force-free magnetic structures which are bounded, energized and finally driven to instability by a differentially rotating dense high-beta atmosphere. The differences are related partly to different drivers (single star, star-star, star-disk, star-cloud) and partly to different magnetic surface fluxes and dimensions (with consequently larger stored and released amounts of energy). Much of the (micro)physics leading to the observed energy partitioning (motion/heating/acceleration/coherent radiation) remains to be sorted out and probably varies greatly going from non-compact single stars to compact accreting stars (role of radiation field, relativistic effects). Finally although radio emission can be obscured by accretion and excretion flows, it appears that the relatively small number of radio active $\mathrm{X}$-ray binaries mirrors intrinsic differences in state of activity, related to the relative mass accretion rate and the stellar magnetic moment.

\section{References}

Adams, F. C., Lada, C. J., and Shu, F. H.: 1987, Astrophys. J. 312, 788.

Alfvén, H.: 1958, Tellus 10, 104. 
Alfvén, H.: 1981, Cosmic Plasma, D. Reidel Publ. Co., Dordrecht, Holland.

Alfvén, H. and Carlqvist, P.: 1967, Solar Phys. 1, 220.

Aly, J.-J.: 1985, Astron. Astrophys. 143, 19.

Aly, J.-J. and Kuijpers, J.: 1989, Astron. Astrophys. (submitted).

André, Ph.: 1986, in T. Montmerle and C. Bertout (eds.), Protostars and Molecular Clouds, CEA, Saclay, France, p. 143.

André, Ph., Montmerle, T., and Feigelson, E. D.: 1987, Astron. J. 93, 1182.

André, Ph., Montmerle, T., Feigelson, E. D., Stine, P. C., and Klein, K.-L.: 1988, Astrophys. J. $335,940$.

Aschwanden, M. J., Hewitt, R. G., and Dulk, G. A.: 1989, in B. M. Haisch and M. Rodonò (eds.), 'Solar and Stellar Flares', presented at IAU Colloq. 104 (unpublished).

Athay, R. G.: 1984, Solar Phys. 93, 123.

Bahcall, J. N., Rosenbluth, M. N., and Kulsrud, R. M.: 1973, Nature Phys. Sci. $243,27$.

Band, D. L. and Grindlay, J. E.: 1984, Astrophys. J. 285, 702.

Bastian, T. S. and Bookbinder, J. A.: 1987, Nature 326, 678.

Bastian, T. S., Dulk, G. A., and Chanmugan, G.: 1985, in R. M. Hjellming and D. M. Gibson (eds.), Radio Stars, D. Reidel Publ. Co., Dordrecht, Holland, p. 225.

Bastian, T. S., Dulk, G. A., and Chanmugan, G.: 1988, Atrophys. J. 324, 431.

Bastian, T. S., Dulk, G. A., and Slee, O. B.: 1988, Astron. J. 95, 794.

Benz, A. O.: 1986, Solar Phys. 104, 99.

Benz, A. O. and Güdel, M.: 1989, in G. Belvedere (ed.), Accretion Disks and Magnetic Fields in Astrophysics, Noto, Sicilia (in press).

Bertout, C., Basri, G., and Bouvier, J.: 1988, Astrophys. J. 330, 350.

Bieging, J. H. and Cohen, M.: 1985, Astrophys. J. 289, L5.

Bieging, J. H., Cohen, M., and Schwartz, P. R.: 1984, Astrophys. J. 282, 699.

Blanken, R. A., Stix, T. F., and Kuckes, A. F.: 1969, Plasma Phys. 11, 945.

Bookbinder, J. A.: 1988, in O. Havnes, B. R. Pettersen, J. H. M. M. Schmitt and J. E. Solheim (eds.), Activity in Cool Star Envelopes, Kluwer, Dordrecht, Holland, p. 257.

Bookbinder, J. A. and Lamb, D. Q.: 1987, Astrophys. J. 323, L131.

Bookbinder, J. A. and Walter, F. M.: 1988, in J. L. Linsky and R. E. Stencel (eds.), Cool Stars, Stellar Systems and the Sun, Fifth Cambridge Workshop, Springer-Verlag, New York, p. 260.

Bookbinder, J. A., Bastian, T. S., Dulk, G. A., and Davis, M. M.: 1989, presented at IAU Colloq. 104 (unpublished).

Borghi, S. and Chiuderi-Drago, F.: 1985, Astron. Astrophys. 143, 226.

Brown, A.: 1987, Astrophys. J. 322, L31.

Brown, A.: 1988, in J. L. Linsky and R. E. Stencel (eds.), Cool Stars, Stellar Systems and the Sun, Fifth Cambridge Workshop, Springer-Verlag, New York, p. 466.

Brown, R. L. and Crane, P. C.: 1979, Astron. J. 83, 1504.

Bührke, T., Brugel, E. W., and Mundt, R.: 1986, Astron. Astrophys. 163, 83.

Butler, C. J., Rodonò, M., Foing, B. H., and Haisch, B. M.: 1986, Nature 321, 679.

Canfield, R. C. and Metcalf, T. R.: 1987, Astrophys. J. 321, 586.

Chanmugan, G.: 1987, Astrophys. Space Sci. 130, 53.

Chanmugan, G. and Dulk, G. A.: 1982, Astrophys. J. 255, L107.

Cohen, M.: 1987, in W. Kundt (ed.), Astrophysical Jets and Their Engines, D. Reidel Publ. Co., Dordrecht, Holland, p. 91.

Cohen, M. and Bieging, J. H.: 1986, Astron. J. 92, 1396.

Davis, R. J., Lovell, B., Palmer, H. P. and Spencer, R. E.: 1978, Nature 273, 644.

DeCampli, W. M. and Baliunas, S. L.: 1979, Astrophys. J. 230, 815.

Dennis, B. R.: 1988, Solar Phys. 118, 49.

Doyle, J. G., Butler, C. J., Byrne, P. B., and van den Oord, G. H. J.: 1988, Astron. Astrophys. $193,229$.

Drake, S. A., Simon, T., and Linsky, J. L.: 1987, Astron. J. 91, 1229.

Drake, S. A., Abbott, D. C., Bastian, T. S., Bieging, J. H., Churchwell, E., Dulk, G. A., and Linsky, J. L.: 1987, Astrophys. J. 322, 902.

Dröge, F.: 1967, Z. Astrophys. 66, 176.

Dulk, G. A.: 1985, Ann. Rev. Astron. Astrophys. 23, 169.

Dulk, G. A.: 1986, Adv. Space Res. 6, 95. 
Dulk, G. A.: 1988, in J. L. Linsky and R. E. Stencel (eds.), Cool Stars, Stellar Systems and the Sun, Fifth Cambridge Workshop, Springer-Verlag, New York, p. 72.

Dulk, G. A., Bastian, T. S., and Chanmugan, G.: 1983, Astrophys. J. 273, 249.

Feigelson, E. D.: 1986, in T. Montmerle and C. Bertout (eds.), Protostars and Molecular Clouds, CEA, Saclay, France, p. 123.

Feigelson, E. D. and Montmerle, T.: 1985, Astrophys. J. 289, L19.

Feldman, P. A.: 1983, in P. B. Byrne and M. Rodonò (eds.), 'Activity in Red-Dwarf Stars', IAU Colloq. 71, 429.

Feldman, P. A., Taylor, A. R., Gregory, P. C., Seaquist, E. R., Balonek, T. J., and Cohen, N. L.: 1978, Astron. J. 83, 1471.

Fürst, E., Benz, A., Hirth, W., Kiplinger, A., and Geffert, M.: 1986, Astron. Astrophys. 154, 377.

Gahm, G. F.: 1986, in P. M. Gondhalekar (ed.), Flares: Solar and Stellar, Rutherford Appleton Lab., U.K.

Gahm, G. F.: 1988, in A. K. Dupree and M. T. V. T. Lago (eds.), Formation and Evolution of Low-Mass Stars, Kluwer, Dordrecht, Holland, p. 295.

Garcia, M. R., Grindlay, J. E., Molnar, L. A., Stella, L., White, N. E., and Seaquist, E. R.: 1988, Astrophys. J. 328, 552.

Gary, D. E.: 1985, in R. M. Hjellming and D. M. Gibson (eds.), Radio Stars, D. Reidel Publ. Co., Dordrecht, Holland, p. 185.

Gary, D. E. and Linsky, J. L.: 1981, Astrophys. J. 250, 284.

Gibson, D. M.: 1983a, in P. B. Byrne and M. Rodonò (eds.), 'Activity in Red-Dwarf Stars', IAU Colloq. 71, 273.

Gibson, D. M.: 1983b, in S. L. Baliunas and L. Hartmann (eds.), Cool Stars, Stellar Systems and the Sun, Springer-Verlag, Berlin, p. 197.

Gibson, D. M.: 1984, in P. F. Gott and P. S. Riherd (eds.), Proc. Southwest Regional Conf. for Astron. Astrophys. No.9, p. 35.

Gibson, D. M.: 1985, in R. M. Hjellming and D. M. Gibson (eds.), Radio Stars. D. Reidel Publ. Co., Dordrecht, Holland, p. 213.

Gibson, D. M. and Fisher, P. L.: 1981, in P. F. Gott and P. S. Riherd (eds.), Proc. Southwest Regional Conf. for Astron. Astrophys. No. 6, p. 33.

Gregory, P. C., Kronberg, P. P., Seaquist, E. R., Hughes, V. A., Woodsworth, A., Viner, M. R., Retallack, D., Hjellming R. M., and Balick, B.: 1972, Nature (Phys. Sci.) 239, 114.

Hamann, F., Simon, M., and Ridgway, S. T.: 1988, Astrophys. J. 326, 859.

Hartmann, L. and Kenyon, S.: 1988, in A. K. Dupree and M. T. V. T. Lago (eds.), Formation and Evolution of Low-Mass Stars, Kluwer, Dordrecht, Holland, p. 163.

Haynes, R. F., Lerche, I., and Murdin, P.: 1980, Astron. Astrophys. 87, 299.

Hénoux, J.-C.: 1987, in V. E. Stepanov and V. N. Obridko (eds.), Solar Maximum Analysis, VNU Science Press, Utrecht, p. 109.

Hershkowitz, N.: 1985, Space Sci. Rev. 41, 351.

Hjellming, R. M.: 1988, in G. L. Verschuur and K. I. Kellermann (eds.), Galactic and Extragalactic Radio Astronomy, Springer-Verlag, Berlin, p. 381.

Hjellming, R. M. and Gibson, D. M.: 1980, in M. R. Kundu and T. E. Gergely (eds.), Radio Physics of the Sun, IAU Colloq. 86, D. Reidel Publ. Co., Dordrecht, Holland, p. 209.

Hjellming, R. M. and Johnston, K. J.: 1985, in R. M. Hjellming and D. M. Gibson (eds.), Radio Stars, D. Reidel Publ. Co., Dordrecht, Holland, p. 309.

Hjellming, R. M. and Johnston, K. J.: 1988, Astrophys. J. 328, 600.

Holman, G. D.: 1983, Adv. Space Res. 2, No. 11, 181.

Holman, G. D.: 1985, Astrophys. J. 293, 584.

Holman, G. D., Eichler, D., and Kundu, M. R.: 1980, in M. R. Kundu and T. E. Gergely (eds.), 'Radio Physics of the Sun', IAU Colloq. 86, 457.

Holman, G. D., Bookbinder, J., and Golub, L.: 1985, in R. M. Hjellming and D. M. Gibson (eds.), Radio Stars, D. Reidel Publ. Co., Dordrecht, Holland, p. 35.

Hughes, V. A. and McLean, B. J.: 1987, Astrophys. J. 313, 263.

Jackson, P. D., Kundu, M. R., and White, S. M.: 1987, Astrophys. J. 316, L85.

Jacobsen, C. and Carlqvist, P.: 1964, Icarus 3, 270.

Johnston, K. J., Spencer, J. H., Simon, R. S. et al.: 1986, Astrophys. J. 309, 707.

Kaitchuk, R. H. and Park, E. A.: 1988, Astrophys. J. 325, 225. 
Kawai, N., Matsuoka, M., Pan, H. C., and Stewart, G. C.: 1989, Publ. Astron. Soc. Japan (in press).

Kellermann, K. I. and Pauliny-Toth, I. I. K.: 1968, Ann. Rev. Astron. Astrophys. 6, 417.

Kenyon, S. J., Hartmann, L., and Hewett, R.: 1988, Astrophys. J. 325, 231.

Klein, K.-L.: 1987, Astron. Astrophys. 183, 341.

Klein, K.-L. and Chiuderi-Drago, F.: 1987, Astron. Astrophys. 175, 179.

Kosugi, T., Dennis, B. R., and Kai, K.: 1988, Astrophys. J. 324, 1118.

Krüger, A.: 1979, Introduction to Solar Radio Astronomy and Radio Physics, D. Reidel, Publ. Co., Dordrecht, Holland.

Kuijpers, J.: 1974, Solar Phys. 36, 157.

Kuijpers, J.: 1975, Astron. Astrophys. 40, 405.

Kuijpers, J.: 1980, in M. R. Kundu and T. E. Gergely (eds.), 'Radio Physics of the Sun', IAU Colloq. 86, 341.

Kuijpers, J.: 1985a, in J. Fanta and G. Pantofliček (eds.), Trends in Physics, Proc. of the Sixth General Conf. of the Europ. Phys. Soc., Prague, p. 170.

Kuijpers, J.: 1985b, in R. M. Hjellming and D. M. Gibson (eds.), Radio Stars, D. Reidel Publ. Co., Dordrecht, Holland, p. 3.

Kuijpers, J. and van der Hulst, J. M.: 1985, Astron. Astrophys. 149, 343.

Kuijpers, J., van der Post, P., and Slottje, C.: 1981, Astron. Astrophys. 103, 331.

Kuijpers, J., Lindberg, L., Raadu, M. A., Stenflo, L., and Torvén, S.: 1989, in preparation.

Kundu, M. R. and Hjellming, R. M.: 1985, in R. M. Hjellming, and D. M. Gibson (eds.), Radio Stars, D. Reidel Publ. Co., Dordrecht, Holland, p. 397.

Kundu, M. R. and Shevgaonkar, R. K.: 1985, Astrophys. J. 297, 644.

Kundu, M. R. and Shevgaonkar, R. K.: 1988, Astrophys. J. 334, 1001.

Kundu, M. R. and Woodgate, B. (eds.): 1986, Energetic Phenomena on the Sun, NASA Conf. Publ. 2439, GSFC, Maryland, Ch. 2, Ch. 5 .

Kundu, M. R., Jackson, P. D., White, S. M., and Melozzi, M.: 1987, Astrophys. J. 312, 822.

Kundu, M. R., Pallavicini, R., White, S. M., and Jackson, P. D.: 1988, Astron. Astrophys. 195, 159.

Lada, C. J.: 1988, in A. K. Dupree and M. T. V. T. Lago (eds.), Formation and Evolution of Low-Mass Stars, Kluwer, Dordrecht, Holland, p. 93.

Lamb, D. Q. and Melia, F.: 1986, in K. O. Mason, M. G. Watson, and N. E. White (eds.), The Physics of Accretion onto Compact Objects, Springer-Verlag, Berlin, p. 113.

Lamb, F. K., Aly, J., Cook, M., and Lamb, D. Q.: 1983, Astrophys. J. 274, L71.

Lang, K. R. and Willson, R. F.: 1986a, Astrophys. J. 302, L17.

Lang, K. R. and Willson, R. F.: 1986b, Astrophys. J. 305, 363.

Lang, K. R. and Willson, R. F.: 1988, Astrophys. J. 326, 300.

Lang, K. R., Bookbinder, J., Golub, L., and Davis, M.: 1983, Astrophys. J. 272, L15.

Lestrade, J.-F.: 1988, in M. J. Reid and J. M. Morgan (eds.), 'The Impact of VLBI on Astrophysics and Geophysics', IAU Symp. 129, 265.

Lestrade, J.-F., Mutel, R. L., Preston, R. A., and Phillips, R. B.: 1988, Astrophys. J. 328, 232.

Lewin, W. H. G., van Paradijs, J., and van der Klis, M.: 1988, Space Sci. Rev. 46, 273.

Lin, R. P., Schwartz, R. A., Kane, S. R., Pelling, R. M., and Hurley, K. C.: 1984, Astrophys. J. 283, 421.

Lindberg, L.: 1987, XVIII Int. Conf. on Phenomena in Ionized Gases, Swansea, U.K.

Lindberg, L.; 1988, Astrophys. Space Sci. 144, 3.

Linsky, J. L. and Gary, D. E.: 1983, Astrophys. J. 274, 776.

Louarn, P., Le Quéau, D., and Roux, A.: 1987, Solar Phys. 111, 201.

Louarn, P., Le Quéau, D., and Roux, A.: 1989, in B. M. Haisch and M. Rodonò (eds.), IAU Colloq. 104, 'Solar and Stellar Flares', Poster Papers, Publ. Catania Astrophys. Obs., Special Volume, p. 329.

Lovell, B.: 1971, Quart. J. Roy. Astr. Soc. 12, 98.

Low, B. C.: 1986, Astrophys. J. 307, 205.

Lu, E. T. and Petrosian, V.: 1988, Astrophys. J. 327, 405.

Lynden-Bell, D. and Pringle, J. E.: 1974, Monthly Notices Roy. Astron. Soc. 168, 603.

Mason, K. O., Cordova, F. A., and White, N. E.: 1986, Astrophys. J. 309, 700.

Massi, M., Felli, M., Pallavicini, R., Tofani, G., Palagi, F., and Catarzi, M.: 1989, Astron. Astrophys. (in press).

McCray, R.: 1966, Science 154, 1320.

McKean, M. E., Winglee, R. M., and Dulk, G. A.: 1989, in B. M. Haisch and M. Rodonò (eds.), IAU Colloq. 104, 'Solar and Stellar Flares', Poster Papers, Publ. Catania Astrophys. Obs., Special Volume, p. 333. 
McLean, D. J. and Labrum, N. R. (eds.): 1985, Solar Radiophysics, Cambridge Univ. Press, Cambridge. Melrose, D. B.: 1973, Australian J. Phys. 26, 229.

Melrose, D. B.: 1986, Instabilities in Space and Laboratory Plasmas, Cambridge Univ. Press, Cambridge.

Melrose, D. B. and Dulk, G. A.: 1982, Astrophys. J. 259, 844.

Melrose, D. B., Hewitt, R. G., and Dulk, G. A.: 1984, J. Geophys. Res. 89, 897.

Mezger, P. G. and Henderson, A. P.: 1967, Astrophys. J. 147, 471.

Molnar, L. A., Reid, M. J., and Grindlay, J. E.: 1984, Nature 310, 662.

Molnar, L. A., Reid, M. J., and Grindlay, J. E.: 1985, in R. M. Hjellming and D. M. Gibson (eds.), Radio Stars, D. Reidel Publ. Co., Dordrecht, Holland, p. 329.

Molnar, L. A., Reid, M. J., and Grindlay, J. E.: 1988a, Astrophys. J. 331, 494.

Molnar, L. A., Reid, M. J., and Grindlay, J. E.: 1988b, in M. J. Reid and J. E. Moran (eds.), 'The Impact of VLBI on Astrophysics and Geophysics', IAU Symp. 129, 279.

Montmerle, T.: 1988, in E. H. Schröter and M. Schüssler (eds.), Solar and Stellar Physics, Springer-Verlag, Berlin, p. 117.

Montmerle, T. and André, Ph.: 1988, in A. K. Dupree and M. T. V. T. Lago (eds.), Formation and Evolution of Low-Mass Stars, Kluwer, Dordrecht, Holland, p. 225.

Morris, D. H. and Mutel, R. L.: 1988, Astron. J. 95, 204.

Mundt, R.: 1988, in A. K. Dupree and M. T. V. T. Lago (eds.), Formation and Evolution of Low-Mass Stars, Kluwer, Dordrecht, Holland, p. 257.

Mutel, R. L. and Lestrade, J.-F.: 1985, Astron. J. 90, 493.

Mutel, R. L. and Weisberg, J. M.: 1978, Astron. J. 83, 1499.

Mutel, R. L., Lestrade, J.-F., Preston, R. A., and Phillips, R. B.: 1985, Astrophys. J. 289, 262.

Mutel, R. L., Morris, D. H., Doiron, D. J. and Lestrade, J.-F.: 1987, Astron. J. 93, 1220.

Nelson, R. F. and Spencer, R. E.: 1988, Monthly Notices Roy. Astron. Soc. 234, 1105.

Newell, R. T., Gibson, D. M., Becker, R. M., and Holt, S. S.: 1980, in P. F. Gott and P. S. Riherd (eds.), Proc. Southwest Regional Conf. for Astron. Astrophys. 5, 13.

Owen, F. N. and Gibson, D. M.: 1978, Astron. J. 83, 1488.

Owen, F. N., Jones, T. W., and Gibson, D. M.: 1976, Astrophys. J. 210, L27.

Papadopoulos, K. and Freund, H. P.: 1979, Space Sci. Rev. 24, 511.

Parker, E. N.: 1988, Astrophys. J. 330, 474.

Penninx, W., Lewin, W. H. G., Zijlstra, A. A., Mitsuda, K., van Paradijs, J., and van der Klis, M.: 1988, Nature 336, 146.

Priedhorsky, W.: 1986, Astrophys. J. 306, L97.

Priedhorsky, W., Hasinger, G., Lewin, W. H. G., Middleditch, J., Parmar, A., Stella, L., and White, N. E.: 1986, Astrophys. J. 306, L91.

Raadu, M. A.: 1984, in R. Schrittwieser and G. Eder (eds.), Proc. Second Symposium on Plasma Double Layers and Topics, Innsbruck, p. 3.

Raadu, M. A. and Rasmussen, J. J.: 1988, Astrophys. Space Sci. 144, 43.

Ramaty, R.: 1969, Astrophys. J. 158, 753.

Rickett, B. J.: 1977, Ann. Rev. Astron. Astrophys. 15, 479.

Rodríguez, L. F., Cantó, J., Torrelles, J. M., and Ho, P. T. P.: 1986, Astrophys. J. 301, L25.

Romney, J. D., Schilizzi, R. T., Fejes, I., and Spencer, R. E.: 1987, Astrophys. J. 321, 822.

Saar, S. H.: 1988, in J. L. Linsky and R. E. Stencel (eds.), Cool Stars, Stellar Systems and the Sun, Fifth Cambridge Workshop, Springer-Verlag, New York, p. 10

Saar, S. H. and Linsky, J. L.: 1985, Astrophys. J. 299, L47.

Schadee, A.: 1986, Adv. Space Res. 6, No. 6, 41.

Schadee, A., de Jager, C., and Švestka, Z.: 1983, Solar Phys. 89, 287.

Seaquist, E. R. and Gregory, P. C.: 1977, Astrophys. Letters 18, 65.

Seaquist, E. R. and Taylor, A. R.: 1985, Astron. J. 90, 2049.

Shklovski, I. S.: 1965, Soviet Astron-AJ 9, 22.

Simon, T., Linsky, J. L., and Schiffer III, F. H.: 1980, Astrophys. J. 239, 911.

Slee, O. B., Haynes, R. F., and Wright, A. E.: 1984, Monthly Notices Roy. Astron. Soc. $208,865$.

Slee, O. B., Touky, I. R., Nelson, G. J., and Renie, C. J.: 1981, Nature 292, 220.

Slee, O. B., Nelson, G. J., Stewart, R. T., Wright, A. E., Innis, J. L., Ryan, S. G., and Vaughan, A. E.: 1987 , Monthly Notices Roy. Astron. Soc. 229, 659.

Slottje, C.: 1978, Nature 275, 520. 
Spangler, S. R.: 1977, Astron. J. 82, 169.

Spangler, S. R., Owen, F. N., and Hulse, R. A.: 1977, Astron. J. 82, 989.

Spencer, R. E., Swinney, R. W., Johnston, K. J., and Hjellming, R. M.: 1986, Astrophys. J. 309, 694

Stähli, M. and Magun, A.: 1986, Solar Phys. 104, 117.

Stepanov, A. V.: 1974, Soviet Astron. 17, 781.

Stepanov, A. V.: 1978, Soviet Astron. Letters 4, 103.

Stewart, R. T., Innis, J. L., Slee, O. B., Nelson, G. J., and Wright, A. E.: 1988, Astron. J. 96, 371.

Stine, P. C., Feigelson, E. D., André, P., and Montmerle, T.: 1988, Astron. J. 96, 1394.

Taylor, A. R. and Gregory, P. C.: 1984, Astrophys. J. 283, 273.

Torvén, S.: 1982, J. Phys. D: Appl. Phys. 15, 1943.

Torvén, S., Lindberg, L., and Carpenter, R. T.: 1985, Plasma Phys. Contr. Fusion 27, 143.

Tsytovich, V. N.: 1973, Ann. Rev. Astron. Astrophys. 11, 363.

Twiss, R. Q.: 1958, Australian J. Phys. 11, 564.

Uchida, Y.: 1986, Adv. Space Res. 6, No. 8, 29.

Uchida, Y. and Sakurai, T.: 1983, in P. B. Byrne and M. Rodonò (eds.), 'Activity in Red-Dwarf Stars', IAU Colloq. 71, 629.

van den Oord, G. H. J.: 1988, Astron. Astrophys. 207, 101.

van den Oord, G. H. J. and Barstow, M. A.: 1988, Astron. Astrophys. 207, 89.

van den Oord, G. H. J. and Mewe, R.: 1989, Astron. Astrophys. (submitted).

van den Oord, G. H. J., Mewe, R., and Brinkman, A. C.: 1989, Astron. Astrophys. (in press).

van den Oord, G. H. J., Kuijpers, J., White, N. E., van der Hulst, J. M., and Culhane, J. L.: 1989, Astron. Astrophys. 209, 296.

van der Laan, H.: 1966, Nature 211, 1131.

Vermeulen, R. C., Schilizzi, R. T., Icke, V., Fejes, I., and Spencer, R. E.: 1988, in M. J. Reid and J. M. Moran (eds.), 'The Impact of VLBI on Astrophysics and Geophysics', IAU Symp. 129, 275.

Vilhu, O., Caillault, J.-P., Neff, J., and Heise, J.: 1988, in O. Havnes, B. R. Pettersen, J. H. M. M. Schmitt, and J. E. Solheim (eds.), Activity in Cool Star Envelopes, Kluwer, Dordrecht, Holland, p. 179.

Wendker, H. J.: 1987, Astron. Astrophys. Suppl. Ser. 69, 87.

White, N. E., Culhane, J. L., Parmar, A. N., Kellett, B. J., Kahn, S., van den Oord, G. H. J., and Kuijpers, J.: 1986, Astrophys. J. 301, 262.

White, S. M., Kundu, M. R., and Jackson, P. D.: 1986, Astrophys. J. 311, 814.

White, S. M., Melrose, D. B., and Dulk, G. A.: 1986, Astrophys. J. 308, 424.

White, S. M., Kundu, M. R., and Jackson, P. D.: 1989, Astrophys. J. (submitted).

Whitehouse, D. R.: 1985, Astron. Astrophys. 145, 449.

Willson, R. F.: 1987, Proc. of Sac Peak Workshop on Coronal Structure and Dynamics (in press).

Willson, R. F. and Lang, K. R.: 1987, Astrophys. J. 312, 278.

Willson, R. F., Lang, K. R., and Foster, P.: 1988, Astron. Astrophys. 199, 255.

Winglee, R. M. and Dulk, G. A.: 1986, Astrophys. J. 307, 808.

Winglee, R. M., Dulk, G. A., and Pritchett, P. L.: 1988, Astrophys. J. 328, 809.

Wu, C. S. and Lee, L. C.: 1979, Astrophys. J. 230, 621.

Zheleznyakov, V. V.: 1967, Soviet Phys. JETP 24, 381.

Zheleznyakov, V. V. and Zlotnik, E. Y.: 1975, Solar Phys. 43, 431. 